\title{
Enteral trickle feeding reduces abnormal intestinal permeability in patients receiving parenteral nutrition
}

\author{
P. G. Lidder ${ }^{1}$, D. Flanagan ${ }^{1}$, S. Fleming ${ }^{2}$, T. Wheatley ${ }^{1}$, J. Rahamin ${ }^{1}$ and S. J. Lewis ${ }^{1}$ \\ ${ }^{1}$ Derriford Hospital, Plymouth, Devon, PL6 8DH, UK and ${ }^{2}$ Royal Cornwall Hospital, Treliske, Truro, Cornwall, \\ TRI $3 L J, U K$
}

Intestinal permeability is altered in inflammatory bowel disease, critical illness, and by nutritional interventions. It has been hypothesised that increased permeability may allow bacterial translocation predisposing the host to systemic sepsis ${ }^{(1)}$. While it is relatively easy to achieve the nutritional targets of patients at risk of malnutrition with total parenteral nutrition (PN), it has been suggested that PN causes intestinal villous atrophy ${ }^{(2)}$ and increased mucosal permeability ${ }^{(3)}$, which may contribute to the higher incidence of septic complications associated with PN compared with enteral nutrition $(\mathrm{EN})^{(4)}$. Unfortunately many hospitalised patients are unable to tolerate or receive their required nutritional intake when delivered by the enteral route (EN) alone. There is little data in human subjects assessing the impact of combining EN and PN.

Thirty elective patients undergoing gastro-oesophagectomy for cancer were assessed on an intention to treat basis within a two-armed randomised trial. Patients with inflammatory bowel disease and diabetes were excluded. One group received $100 \%$ of their nutritional requirements with PN. The second group achieved their target with a combination of $70 \%$ PN and $30 \%$ EN. Feeding regimes were isocaloric and isonitrogenous and administered on a continuous basis over $20 \mathrm{~h}$ with a $4 \mathrm{~h}$ break. Non-steroidal inflammatory drugs (NSAIDs) were excluded from post-operative analgesic regimes. Intestinal permeability was measured using the differential absorption of a mannitol/lactulose solution administered through a surgically placed feeding jejunostomy. White cell count (WCC) and C-reactive protein (CRP) were recorded as were complication rates, patient demographics and length of hospital stay.

Both groups were well matched demographically, with no significant differences in operative duration, APACHE II physiological scores or caloric intake. Following surgery, intestinal permeability increased from the preoperative baseline measurement. However, in patients receiving a combination of EN and PN there was an earlier improvement with reductions occurring on day 2 , rather than day 3 (PN group). These differences were significant $(P<0.001)$. CRP and WCC were not different between the groups and demonstrated peaks at day 2. Intestinal permeability positively correlated with other biochemical markers of inflammation $(\mathrm{CRP}: r=0.19, P=0.034$; WCC: $r=0.26, P=0.01)$. There were no significant differences in morbidity and no mortality.

Enteral feeding is the preferred route of nutritional support in critically ill patients. However, particularly in surgical patients there may be restrictions due to the surgery or intolerance of adequate feed volume. Trickle feeding with enteral nutrition in conjunction with parenteral feed was well tolerated and facilitated a rapid restoration of intestinal permeability compared with PN alone. This study was underpowered to detect clinical effects but there were no significant differences in morbidity, mortality or length of hospital stay. A large randomised clinical trial may demonstrate clinical benefit through the maintenance of normal gut integrity.

1. Deitch EA, Kemper AC, Specian RD et al. (1992) J Trauma 32, 141-147.

2. Sano Y, Gomez FE, Kang W, et al. (2007) Parenter Enteral Nutr 31, 351-356.

3. Deitch EA (1994) Gut 35, S23-S27.

4. Moore FA, Moore EE, Jones TN et al. (1989) J Trauma 29, 916-922. 